\title{
Ephemeropteren und Plecopteren aus bulgarischen Höhlen
}

\author{
Von Boris K. Russevi)
}

Mit Tafel 41(1)

Im Katalog für Höhlentiere teilt Wolf (1937) nur drei Arten Eintagsfliegen mit, die in Höhlen aufgefunden wurden, und zwar Baëtis bioculatus (L.), Habrophlebia fusca (Curtis) und Ephemera danica O. F. Müller (die erste Art in rumänischen, die übrigen zwei in französischen Höhlen aufgefunden). In obenerwähntem Katalog wird überhaupt nichts über aufgefundene Plecopteren-Arten in den Höhlen mitgeteilt.

Franciscolo (1955, S. 148) findet die Eintagsfliege Habrophlebia fusca (Curtis) im ,Tana de l'Orpe", und Torii (1964, S. 126) teilt die Steinfliege Nemoura sp. für die japanischen Höhlen mit.

In seiner Arbeit über die Neuropterenfauna Bulgariens teilt Buresch (1936) die Arten Ecdyonurus venosus (Fabr.) (Ephem.) und Perla marginata bureschi Schönemund (Plecopt.) mit, die von ihm in der Wasserhöhle Cerovo längs dem Isker-Durchbruch angetroffen wurden. Eine erhebliche Anzahl von Imagines der ersten Art wurden von den Höhlenwänden gesammelt, ungefähr 2 bis $20 \mathrm{~m}$ vom Eingang entfernt. Larven der zweiterwähnten Art wurden aus dem Bach sogar bis $150 \mathrm{~m}$ Entfernung vom Höhleneingang aufgesammelt.

Damit sind alle Auskünfte über das Auffinden von Eintagsfliegen und Steinfliegen in den bulgarischen Höhlen erschöpft²).

Die dieser Mitteilung zugrunde liegenden Materialien wurden während häufiger Studien der bulgarischen Höhlen gesammelt. Das Material wurde mir von den Kollegen V. Guéorguiev, St. Andreev und An. Petrova liebenswürdigerweise zur Verfügung gestellt, wofür ich ihnen an dieser Stelle meine Erkenntlichkeit aussprechen will. Besonderen Dank schulde ich dem Kollegen V. Guéorguiev für die Hilfe, die er mir beim Ausfindigmachen der entsprechenden Literatur angedeihen ließ.

\footnotetext{
1) Zoologisches Institut der Bulgarischen Akademie der Wissenschaften. Boul. Russki n' I Sofia/Bulgarien.

2) Verschiedene Daten über diese Höhle werden in der Monographie von Guéorguiev et Beron (1962, S. 373) gegeben.
} 


\section{Ordnung: EPHEMEROPTE RA}

Unterordnung: Leptophlebioidea

Habroleptoides modesta (Hagen) 1864

Fundort: Bach in der Höhle,,Balabanova dupka“, Dorf Komštica ${ }^{3}$ ), leg. Vl. Beškov, 15. X.1960.

Material: 1 Larve.

Verbreitung: Fast ganz Europa.

Paraleptophlebia tumida Bengtss. 1930

Fundort: Bach in der Höhle ,Parnicite“ beim Dorfe Bežanovo, Kreis Loveč, leg. An. Petrova, 2. VI.1965.

Beschreibung: 12 Larven, ca. $8 \mathrm{~mm}$. Das erste Kiemenpaar ist ein wenig kleiner als die übrigen. Die Maxillarpalpen sind verhältnismäßig klein und nicht genügend dicht behaart. Die Dornen auf der Unterseite der Hinterschenkel sind zugespitzt, einige unter diesen sind kurz. Die Zähne nehmen ungefähr $3 / 4$ der Krallenlänge ein (Figs. 1-5) (cf. Macan, 1961, S. 26-27).

Verbreitung: England, Norwegen, Schweden, Finnland, Tschechoslowakei, Bulgarien (Russev, 1964, S. 20).

Paraleptophlebia sp.

Fundort: Höhle „Parnicite“ beim Dorf Bežanovo, Kreis Loveč, leg. St. Andreev, 5. VI.1965.

Beschreibung: 1우 Subimago. Körperlänge: $8,5 \mathrm{~mm}$, Länge der Schwanzborsten: $9 \mathrm{~mm}$, Länge des ersten Flügelpaares: $8 \mathrm{~mm}$.

\section{Ordnung: PLECOPTERA \\ Unterordnung: Filipalpia \\ Familie: Nemouridae}

\section{Nemoura sp.}

Fundort: Bach in der Höhle ,Dolnata vodna peštera" beim Dorfe Želen-Bov, unterhalb der Höhle ,Mečata dupka“. 900 m über dem Wasserspiegel, leg. V. Guéorguiev, 20. VI.1958.

Material: 1 Larve, $7 \mathrm{~mm}$.

Bemerkung i Das Larvenstadium dieser Gattung kann noch immer nicht mit Sicherheit bis zur Art bestimmt werden (Illies, 1955, S. 130).

$\left.{ }^{3}\right)$ Auskünfte über die Höhle werden von Guéorguiev et Beron (1962, S. 358) gegeben. 


\section{Unterordnung: Setipalpia}

Familie: Perlidae

\section{Dinocras cephalotes (Curtis) 1827}

Fundort: Höhle ,Kumanica“, Neškovci, Dorf Černi Ossǎm bei Sdt. Trojan, leg. St. Andreev, 23. VII.1962.

Beschreibung: oત 17 mm, kurzflügelig, Fortsatz des Tergits 10 einfach (cf. Illies, 1955, S. 105-107).

Verbreitung: Schweiz, Deutschland, England, Österreich, Belgien, Dänemark, Spanien, Frankreich, Niederlande, Italien, Norwegen, Rumänien, Schweden, Tschechoslowakei (Aubert, 1946), Bulgarien (Buresch, 1936), UdSSR - europäischer Teil (Zhiltzova, 1964), Polen (Wojtas, 1964). Wird häufig in verschiedenen Gewässern angetroffen: von Bächen bis zu großen Flüssen, und in Nordeuropa auch in Seen (Aubert, 1954).

Die in den bulgarischen Höhlen aufgefundenen drei Eintagsfliegen nud zwei Steinfliegen sind trogloxen.

\section{ZUSAMMENFASSUNG}

In den bulgarischen Höhlen wurden bis jetzt nur die Eintagsfliege Ecdyonurus venosus (Fabr.) und die Steinfliege Perla marginata bureschi (Schoenemund) angetroffen. Der Autor teilt folgende Arten mit, die von seinen Kollegen in den bulgarischen Höhlen gesammelt wurden: Ephemeroptera - Habroleptoides modesta (Hagen), Paraleptophlebia tumida Bengtss. und Paraleptophlebia sp.; Plecoptera - Nemoura sp. und Dinocras cephalotes (Curtis). Alle mitgeteilten Arten sind trogloxen.

\section{SUMMARY}

The mayfly Ecdyonurus venosus (Fabr.) and the stonefly Perla marginata bureschi (Schoenemund) have been previously recorded from Bulgarian caves. The author has found the following additional species in the material collected by his colleagues in Bulgarian caves: Ephemeroptera - Habroleptoides modesta (Hagen), Paraleptophlebia tumida Bengtss. and Paraleptophlebia sp.; Plecoptera - Nemoura sp. and Dinocras cephalotes (Curtis). All these species are trogloxenes.

\section{LITERATUR}

Aubert, J. (1946) - Les Plécoptères de la Suisse Romande. Mitt. Schweiz. Ent. Ges. 20: 7-128.

- (1954) - Dinocras klapaleki n.sp. et la répartition des Dinocras en Suisse et en Europe (Plécoptères Perlidae). Mitt. Schweiz. Ent. Ges. 27: 437-440. 
Buresch, Iw. (1936) - Beitrag zum Studium der Neuropterenfauna Bulgariens (Insecta, Neuroptera), Mitteil. Bulg. Entom. Ges. 9: 135-150. Sofia.

Franciscolo, M. (1955) - Fauna cavernicola del Savonesa. Ann. Mus. Civ. Stor. Natur. Giacomo Doria. 67: 1-223.

Guéorguiev, V., et Beron, P. (1962) - Essai sur la faune cavernicole de Bulgarie. Ann. Spéléol. 17: 285-441.

Illies, J. (1955) - Steinfliegen oder Plecoptera, in Dahl's Tierwelt Deutschlands. Gustav Fischer, Jena 43: 1-150.

Macan, T. T. (1961) - A Key to the Nymphs of the British Species of Ephemeroptera. Freshwater Biol. Assoc. 20:1-64.

Russev, B. (1964) - Hydrobiologische Untersuchungen der Arda und einiger ihrer Nebenflüsse. Bull. de l'Inst. Zool. et Mus. Acad. Bulg. Sci. 17: 5-49.

ToriI, H. S. (1964) - Katalog der Höhlentiere in Japan und seinen Nachbarländern. Dritter Intern. Kongreß für Speläologie. 3: 113-138.

Wojtas, Fr. (1964) - Die Plecopterenfauna Polens mit besonderer Berücksichtigung des Tatragebiets. Verh. 3. intern. Symp. Plecopteren.

Wolf, B. (1937) - Animalium Cavernarum Catalogus. 3: 65-144.

Zhiltzova, L. A. (1964) - Die Plecopteren des europäischen Teiles der Sowjetunion und des Kaukasus. Verh. 3. intern. Symp. Plecopteren.

\section{ERKLÄRUNG DER TAFEL 41 (1)}

Fig. 1: Paraleptophlebia tumida - Maxillarpalpe. Fig. 2: Paraleptophlebia tumida - Dornen auf der Unterseite der Hinterschenkel. Fig. 3: Paraleptophlebia tumida - Kralle des Hinterbeines. Fig. 4: Paraleptophlebia tumida - I. Paar Tracheenkiemen. Fig. 5: Paraleptophlebia tumida - II. Paar Tracheenkiemen. 


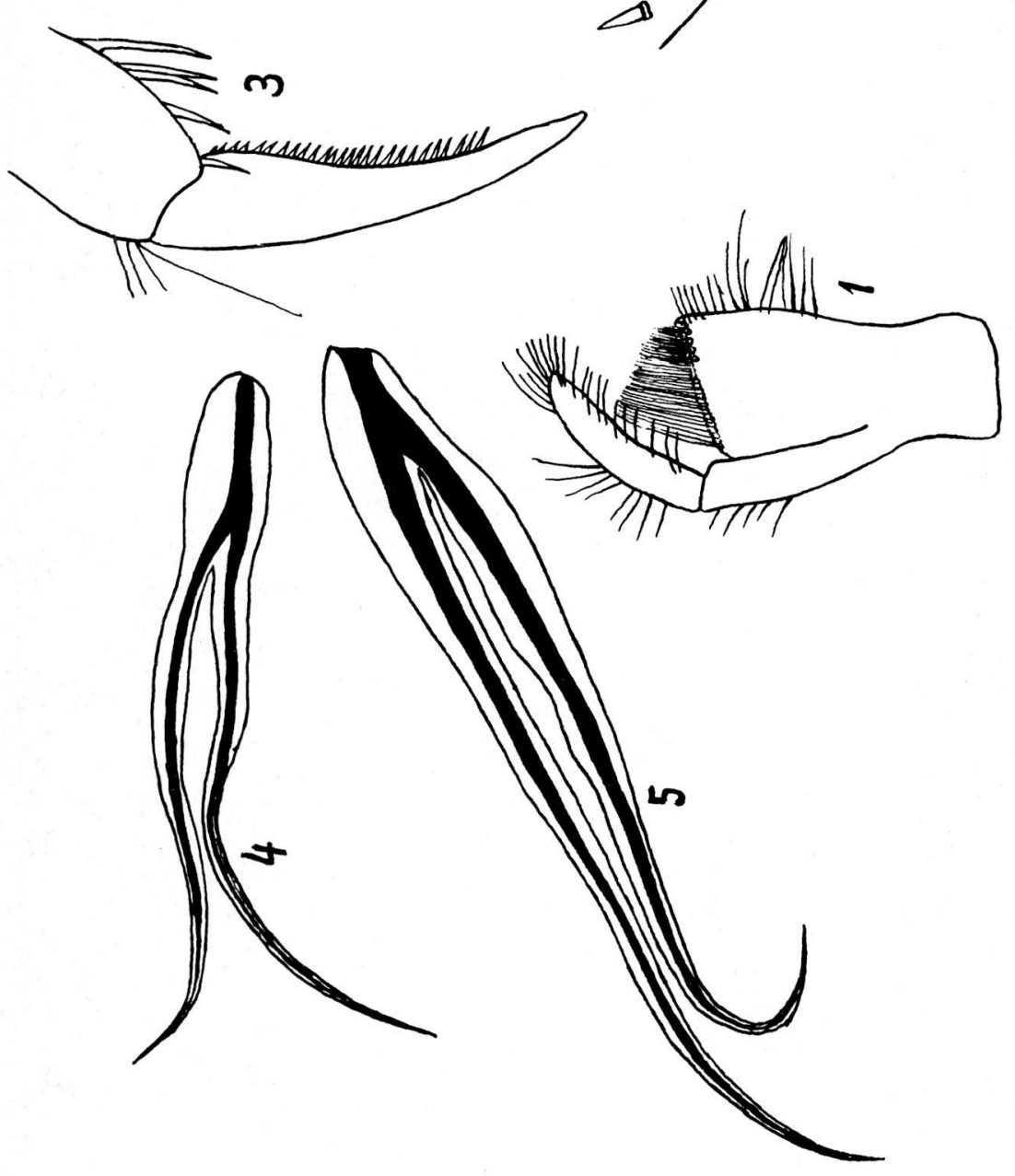

